\title{
Impact of Correlation of Predictors on Discrimination of Risk Models in Development and External Populations
}

\author{
Suman Kundu ${ }^{1^{*}}$, Madhu Mazumdar ${ }^{2}$ and Bart Ferket ${ }^{2}$
}

${ }^{1}$ Division of Cardiology, Department of Medicine, Vanderbilt University Medical Center, Nashville, USA

${ }^{2}$ Institute for Healthcare Delivery Science, Department of Population Health Science and Policy, Icahn School of Medicine at Mount Sinai, New York, USA

*Corresponding author: Kundu S, Department of Medicine, Vanderbilt University Medical Center, Nashville, USA, Tel: +1-404-924-0306; Fax: +1-615-322-3837; E-mail: suman.kundu@vanderbilt.edu

Received date: Nov 17, 2016, Accepted date: Nov 19, 2016, Published date: Nov 23, 2016

Copyright: (c) 2016 Kundu S, et al. This is an open-access article distributed under the terms of the Creative Commons Attribution License, which permits unrestricted use, distribution, and reproduction in any medium, provided the original author and source are credited.

Citation: Kundu S, Mazumdar M, Ferket B (2016) Impact of Correlation of Predictors on Discrimination of Risk Models in Development and External Populations. J Biom Biostat 7: 320. doi:10.4172/2155-6180.1000320

\section{Correlation of Predictors on Discrimination of Risk Models}

Prediction models to estimate disease risk and identify individuals at high risk are widely advocated for optimizing prevention and management of multifactorial diseases. For several common complex diseases including different forms of cancer, diabetes, and cardiovascular disease, a number of prediction models have been developed in various populations. The predictive performance of these risk models is typically assessed by evaluating discrimination which is the ability of the model to separate those with events from those without events. After developing a risk model, it is essential to investigate the model's discriminative performance within an external population to judge about the generalizability and reliability of the risk model. A risk model that does not have an appreciable predictive ability in an external population may have limited opportunity to investigate further, aiming implementation in practice.

It is commonly observed that when a prediction model is validated within an external population, the discriminative ability expressed by the area under the receiver operating characteristic curve (AUC) decreases. However sometimes the AUC is able to increase as observed in number of validation studies. Several studies have shown how the
AUC is impacted by a different case-mix within the validation sample, and heterogeneity in the effect sizes of risk factors among development and validation samples. Using simulation studies, we investigated the impact of correlation among predictors on the AUC in the development sample and how correlation should be interpreted in the light of the other parameters such as the distributions of predictors among cases and controls, and different strengths of predictive effects that may attenuate the AUC in external populations.

Simulation results showed that the AUC depends on the correlation among the predictors in the development and external validation sample. Increasingly negative correlations improve the AUC. The AUC in external populations can be either lower or higher as compared to the AUC estimated in the derivation cohort. This suggests, although the AUC estimated in the derivation sample is not promising, the same risk model can have a promising AUC at external validation. Conversely, even though the AUC in both derivation and a particular validation dataset is high, the same risk model can perform poorly in another external population. Since there may be heterogeneity in correlation structures across various populations and among cases and controls, the predictive ability of risk models should be assessed in different external populations in order to ascertain generalizability. 